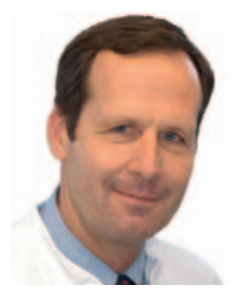

Univ.-Prof. Dr. Jörg C. Prinz

joerg.prinz@med.uni-muenchen.de

Der Themenschwerpunkt «Psoriasis» der aktuellen Ausgabe von Karger Kompass Dermatologie mag dem einen oder anderen ermüdend erscheinen, da Psoriasis in den letzten Jahren eine hohe Präsenz in den dermatologischen Fachmedien hatte. Dennoch ist der Themenschwerpunkt mehr als berechtigt, da sowohl die Erkenntnisse über die Psoriasispathogenese als auch die Entwicklung neuer Therapieformen rasant fortschreiten und so dem Dermatologen einen steten Weiterbildungsdruck bescheren. Wie keine andere Erkrankung in der Dermatologie unterliegt die Psoriasis - neben dem malignen Melanom - seit 10 bis 20 Jahren einem revolutionären Wandel im Verständnis und in den Behandlungsmöglichkeiten. Hieraus ergeben sich zahlreiche spezifische Fragestellungen im Umgang mit der Erkrankung, der neben dem Wissen auch klinische Erfahrung voraussetzt, getreu der Aussage Albert Einsteins «Wissen ist Erfahrung, alles andere ist Information».

Eine grundsätzliche Neuerung hat das Verständnis der Psoriasispathogenese erlebt. Aktuelle Daten zeigen, dass Psoriasis vulgaris auf einer Autoimmunreaktion gegen Melanozyten beruht, die präferentiell durch das Hauptrisikoallel, HLA-C ${ }^{*} 06: 02$, vermittelt wird [1]. Hierbei präsentiert $\mathrm{HLA}-\mathrm{C}^{*} 06$ : 02, aber auch andere Moleküle aus der

\title{
Psoriasis - der Wandel des Beständigen
}

Gruppe der humanen Leukozyten Klasse-IAntigene (HLA-Klasse I), melanozytäre Autoantigene an das Immunsystem. Diese werden von CD8 ${ }^{+}$T-Zellen erkannt und vermitteln so die T-Zell-Aktivierung in der Haut. Die daraus folgende T-Zellantwort wird damit zur Triebkraft der psoriatischen Entzündung. Sie ist durch ein bestimmtes Zytokinmuster gekennzeichnet, das als $T_{H} 17$ oder $T_{C / S} 17$ bezeichnet wird, je nachdem, ob diese Zytokine von CD4+ Helfer-TZellen oder von $\mathrm{CD}^{+}$cytotoxischen/Suppressor-T-Zellen produziert werden. Die Zahl 17 bezieht sich hierbei auf die Interleukine -17A, -17A/F und -17F, die dieses Zytokinmuster von anderen Zytokinmustern unterscheiden. Eigentlich dienen T-Zellen vom $T_{H} 17$ oder $T_{C / S} 17-T y p$ der antimikrobiellen Immunabwehr. Ihre physiologische Aufgabe besteht in der Abwehr von Infektionen, die durch Bakterien oder Pilze entstehen. Werden sie aber im Rahmen einer pathogenen, d.h. krankmachenden Immunantwort aktiviert, vermitteln sie chronische Entzündungen. Hiermit werden auch die neueren therapeutischen Ansätze plausibel: Antagonisten des Tumornekrose-Faktors a (TNF-a) hemmen die Aktivierung und Effektoraktivitäten der pathogenen Immunantwort, Antikörper gegen IL-17 neutralisieren das Haupteffektor-Zytokin und Antikörper gegen IL-12 und IL-23 blockieren die Aktivierung und Differenzierung der $T_{H} 17$ oder $T_{C / S} 17$ T-Zellen, sodass die pathogene Immunantwort zumindest vorübergehend unterbunden wird.

In der vorliegenden Ausgabe des Karger Kompass Dermatologie werden verschiedene Aspekte der Psoriasis aufgegriffen, die sich mit Mundschleimhautveränderungen, ku- tanen Begleiterkrankungen und der Therapie der Psoriasis beschäftigen. Neben der Psoriasis als Kernthema dieser Ausgabe werden als weitere dermatologisch wichtige Themen die Notwendigkeit zur Prävention aktinischer Keratosen sowie eine bessere Aufklärung zum UV-Schutzverhalten diskutiert, ebenso die Wirkung von Ketokonazol bei der seborrhoischen Dermatitis der Kopfhaut und des Peptidantibiotikums Tyrothricin bei Akne vulgaris. Die Prognose von Nahrungsmittelallergien im Kindesalter sowie die Rolle von Zeckenbissen bei der Auslösung der chronischen Urtikaria stellen spannende allergologische Aspekte in diesem Heft dar. Als interessierter Leser können sie sich zudem über faziale Lipodystrophie bei HIV-Therapie, Honigapplikation bei der Wundheilung sowie den Stellenwert von Dapson in der Behandlung des kutanen Lupus erythematodes informieren.

Diese Auswahl von aktuellen Beiträgen bietet gemeinsam mit den zugehörigen Kommentaren als Verbindung von Neuwissen und Erfahrung ein praktisch relevantes Spektrum von interessanten Themen. Ihnen wünsche ich beim Lesen ebenso viel Spaß und Anregung, wie ich sie bei ihrer Durchsicht hatte.

Herzlichst,

Ihr

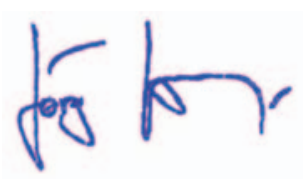

\section{Referenzen}

1 Arakawa A, Siewert K, Stöhr J, et al.: Melanocyte antigen triggers autoimmunity in human psoriasis. J Exp Med 2015;212:2203-2212.

\section{KARGER}

Fax +4976145207 14

information@karger.com

www.karger.com
(C) 2016 S. Karger GmbH, Freiburg

2296-5424/16/0042-0065\$39.50/0

Accessible online at:

www.karger.com/kkd
Univ.-Prof. Dr. Jörg C. Prinz

Klinik und Poliklinik für Dermatologie und Allergologie, Klinikum der Universität München Ludwig-Maximilians-Universität

Frauenlobstraße 9-11

80337 München, Deutschland 